\title{
Comparison of the effects of etomidate, ketamine, sodium thiopental, and midazolam on the mortality of patients with COVID-19 requiring intubation
}

\author{
Abbas Edalatkhah ${ }^{1}$, Mohammad Reza Kazemi $^{*}{ }^{\mathbb{D}}$, Fatemeh Samadi Khorshidi ${ }^{1}$, Zohreh Akhoundimeybodi $^{2}$, Seyed $^{2}$ \\ Mohsen Seyedhosseini ${ }^{3}$, Soheila Rostami ${ }^{3}$, Bibi Vaghihe Hosseini ${ }^{3}$, Zohreh Akhondi ${ }^{3}$, Yaser Ghelmani ${ }^{4}$
}

Received: 25 Jun 2020

Published: 15 Apr 2021

\section{Abstract}

Background: Among the medications administered for the management of COVID-19 patients, the induction drugs used for intubation have received little attention. The aim of this study was to compare the effect of induction drugs on the mortality of patients with COVID-19 requiring intubation.

Methods: In this retrospective study, all patients who were admitted to Shahid Sadoughi and Shahid Rahnemoun hospitals in Yazd from February to March 2020 with definitive diagnosis of COVID-19 and needed intubation were enrolled. Patients were divided into 4 groups based on the type of drugs used in intubation, and mortality rate was assessed at the end of the first, second, fourth, and seventh days of the study. Statistical analyses were performed using SPSS 20 and P values $<.05$ was considered significant.

Results: In this study, 76 patients were examined. Patients were divided into 4 groups, of which 21 were in etomidate group, 8 in ketamine group, 21 in sodium thiopental group, and 35 in midazolam group. Mortality rate in these 4 groups was $25 \%, 12.5 \%, 14.3 \%$, and $14.3 \%(p=0.822)$, respectively at the end of the first day after intubation; it was $83.3 \%, 12.5 \%, 28.6 \%$, and $25.7 \%(p=0.001)$, respectively, at the end of the second day; it was $83.3 \%, 12.5 \%, 42.9 \%$, and $42.9 \%$ ( $\mathrm{p}=0.015$ ), respectively, until the end of the fourth day; it was $100 \%, 25 \%, 61.9 \%$, and $65.7 \%(\mathrm{p}=0.007)$, respectively, until the end of the seventh day. Admission to intubation time interval was $0.91 \pm 0.99,3.12 \pm 1.95,4.09 \pm 2.44$, and $4.74 \pm 2.62$ days, respectively $(\mathrm{p}<0.001)$.

Conclusion: The results of this study suggest that the use of etomidate may be associated with higher mortality in COVID-19 patients. Further studies are needed to verify the results of this study.

Keywords: Mortality, COVID-19, Intubation, Etomidate

Conflicts of Interest: None declared

Funding: This study was funded by the officials of Shahid Sadoughi Hospital.

*This work has been published under CC BY-NC-SA 1.0 license.

Copyright $₫$ Iran University of Medical Sciences

Cite this article as: Edalatkhah A, Kazemi MR, Samadi Khorshidi F, Akhoundimeybodi Z, Seyedhosseini SM, Rostami S, Hosseini BV, Akhondi Z, Ghelmani Y. Comparison of the effects of etomidate, ketamine, sodium thiopental, and midazolam on the mortality of patients with COVID-19 requiring intubation. Med J Islam Repub Iran. 2021 (15 Apr);35:49. https://doi.org/10.47176/mjiri.35.49

\section{Introduction}

Airway management has been one of the biggest challenges in patients with COVID-19 since the beginning of the epidemic. The use of noninvasive methods, such as high-flow nasal cannula and noninvasive ventilation, has

Corresponding author: Dr Mohammad Reza Kazemi, kazemim360@gmail.com

1. Department of Emergency Medicine, Faculty of Medicine, Shahid Sadoghi University of Medical Science, Yazd, Iran

2. Department of infectious diseases, Faculty of Medicine, Shahid Sadoghi University of Medical Sciences, Yazd, Iran

3. Intensive Care Unit, Nursing Faculty, Shahid Sadoughi University of Medical Sciences, Yazd, Iran

4. Clinical Research Development Center of Shahid Sadoughi Hospital. Shahid Sadoghi University of Medical Sciences, Yazd, Iran been associated with a high risk of transmitting the disease through aerosols despite the fact that a great number of the affected patients require intubation (1). The biggest challenge about the management of the intubation process in

$\uparrow$ What is "already known" in this topic:

Induction drugs are one of the essential drugs used in intubation and its selection should be made by considering the complications of the drug and patients' clinical condition. However, the researchers have given less attention to the drugs used for the intubation of COVID-19 patients.

$\rightarrow$ What this article adds:

This study revealed that the use of etomidate for intubation of COVID-19 patients can be associated with a higher risk of mortality. 
these patients has been always the identification of ways to maintain the safety and health of the treatment staff without the slightest inter ruption in patient treatment .The researchers have given less attention to the induction drugs used for the intubation of COVID-19 patients with regard to their efficacy and complications.

However, patients with COVID-19 are often elderly and have multiple comorbidities, such as hypertension and diabetes (2). The critically ill patients reportedly have a high mortality rate; moreover, $49 \%$ to $67 \%$ of the patients need to be admitted to intensive care units (3-5), and even up to $97 \%$ of them require invasive intubation (6) .The most common causes of mortality in COVID-19 patients, include respiratory failure $(63 \%)$, shock $(7 \%)$, respiratory failure+shock (33\%), and unknown causes (7\%) (7).

Induction agents constitute an important category of drugs used for intubation. To the best of our knowledge, no previous studies have examined the possible relationship between the drugs used in intubation of COVID-19 patients and their mortality. Thus, the present study aimed to investigate the associations of etomidate, ketamine, sodium thiopental, and midazolam, as induction agents used for intubation, with the in-hospital mortality of COVID-19 patients.

\section{Methods}

This retrospective study was conducted on all patients who were admitted to Shahid Sadoughi and Shahid Rahnemoun hospitals in Yazd, Iran, with a definitive diagnosis of COVID-19 and needed intubation from February to March 2020. The definitive diagnosis of the patients had been established based on the reverse transcriptionpolymerase chain reaction assay.

The demographic and medical information of the patients was collected from medical records by a people who had no role in patient management. The collected demographic information included age, gender, and history of previous illnesses. The recorded medical data were related to the preintubation conditions of patients, including blood pressure, oxygen saturation, heart rate, white blood cell (WBC,) and lymphocyte count, BUN, creatinine, sodium $(\mathrm{Na})$, potassium $(\mathrm{K})$, Glasco coma scale (GCS), $\mathrm{PH}$ admission to intubation interval, type of drug used for intubation, sequential organ failure assessment (SOFA) score, and the Acute Physiology and Chronic Health Evaluation II (APACHEII) score. The exclusion criteria were (1) information inadequacy; (2) crush intubation; and (3) use of induction drugs other than etomidate, ketamine, sodium thiopental, or midazolam. Finally, the mortality percent was compared among the groups at the end of the first, second, fourth, and seventh days of the study.

\section{Statistical Analysis}

All statistical analyses were performed by Statistical Package for Social Sciences (SPSS) software 20th release, using Kruskal Wallis test, One-way ANOVA, and chi square tests. Two tailed $\mathrm{P}$ values $<.05$ was considered significant.

\section{Results}

A total of 103 patients were nominated for the study; however, 27 cases were excluded from the study due to information inadequacy $(n=8)$, crush intubation $(n=7)$, and use of drugs other than ketamine, etomidate, sodium thiopental, or midazolam $(n=12)$. Finally, 76 patients were included in the final data analysis, out of whom 47 $(61.8 \%)$ were male, and the mean age of the patients was $64.39 \pm 18.87$ (13-94) years (Table 1).

Depending on the type of drug used for induction, the patients were divided into 4 groups of etomidate $(n=12)$, ketamine $(n=8)$, sodium thiopental $(n=21)$, and midazolam $(n=35)$. Hypoxia was present in most of the patients $(n=58 ; 76.3 \%)$ before intubation. Furthermore, $32(42.1 \%)$ patients had tachycardia with a rate above 100 beats per minute, and $9(11.8 \%)$ had hypotension with a MAP of less than $70 \mathrm{~mm} \mathrm{Hg}$.

The results revealed no significant difference among the 4 groups in terms of age, gender distribution, blood pres-

Table 1. Comparison of the age, gender, past medical history and pre-intubation heart rate, mean arterial pressure and oxygen saturation

\begin{tabular}{|c|c|c|c|c|c|}
\hline Characteristic & $\begin{array}{c}\text { Etomidate } \\
\mathrm{N}=12\end{array}$ & $\begin{array}{l}\text { Ketamine } \\
\mathrm{N}=8\end{array}$ & $\begin{array}{l}\text { Sodium thiopental } \\
\mathrm{N}=21\end{array}$ & $\begin{array}{c}\text { Midazolam } \\
\mathrm{N}=35\end{array}$ & $\mathrm{p}$ \\
\hline Age (years) & $63.8 \pm 19.8$ & $60.4 \pm 23.1$ & $60.3 \pm 19.4$ & $68 \pm 17.3$ & $0.477^{*}$ \\
\hline Heart rate $($ beat $/ \mathrm{min})$ & $103.6 \pm 20.9$ & $90.1 \pm 14.7$ & $106.1 \pm 22.2$ & $94.9 \pm 18$ & $<0.001^{*}$ \\
\hline Mean arterial pressure $(\mathrm{mmHg})$ & $76.8 \pm 9.4$ & $86.3 \pm 14.8$ & $87.1 \pm 12.6$ & $87.2 \pm 18.6$ & $0.094 * *$ \\
\hline Oxygen Saturation & $66.4 \pm 13.1$ & $74.25 \pm 11.4$ & $76.6 \pm 12.3$ & $84 \pm 12.7$ & $0.224 * *$ \\
\hline Male gender & $7(58.3 \%)$ & $7(87.5 \%)$ & $11(52.4 \%)$ & $22(62.9 \%)$ & $0.382 * * *$ \\
\hline \multicolumn{6}{|l|}{ Number (percent) } \\
\hline Positive PMH Number (percent) & $7(58.3 \%)$ & $5(62.5 \%)$ & $18(85.7 \%)$ & $33(94.3 \%)$ & $0.014 * * *$ \\
\hline $\mathrm{WBC} \times 109 / \mathrm{L}$ & $7.78 \pm 2.42$ & $7.78 \pm 2.4$ & $7.83 \pm 3.6$ & $6.93 \pm 0.35$ & $0.673 * *$ \\
\hline Lymphocyte count $\times 109 / \mathrm{L}$ & $1.1 \pm 0.8$ & $1.4 \pm 1.8$ & $1.13 \pm 1.1$ & $1.31 \pm 1.08$ & $0.497^{*}$ \\
\hline Bun & $34.5 \pm 25.3$ & $17.12 \pm 4.22$ & $21.47 \pm 10.95$ & $21.6 \pm 12.09$ & $0.448^{*}$ \\
\hline $\mathrm{Cr}$ & $2.33 \pm 2.07$ & $1.11 \pm 0.33$ & $1.45 \pm 0.91$ & $1.71 \pm 1.27$ & $0.333^{*}$ \\
\hline $\mathrm{Na}$ & $144.5 \pm 10.4$ & $135.5 \pm 6.45$ & $137.7 \pm 6.48$ & $137.05 \pm 5.58$ & $0.009 * *$ \\
\hline $\mathrm{K}$ & $4.05 \pm 0.7$ & $4.6 \pm 1.46$ & $3.9 \pm 7.8$ & $3.91 \pm 0.6$ & $0.155^{* *}$ \\
\hline $\mathrm{PH}$ & $7.37 \pm 0.14$ & $7.2 \pm 0.16$ & $7.37 \pm 0.14$ & $7.32 \pm 0.16$ & $0.115^{*}$ \\
\hline GCS & $10.66 \pm 1.96$ & $10.87 \pm 2.1$ & $10.9 \pm 1.87$ & $11.31 \pm 2.11$ & $0.801 *$ \\
\hline Admit to intubation interval (days) & $0.91 \pm 0.99$ & $3.12 \pm 1.95$ & $4.09 \pm 2.44$ & $4.74 \pm 2.62$ & $<0.001^{*}$ \\
\hline SOFA score & $3.12 \pm 2.1$ & $2.8 \pm 2$ & $3.23 \pm 1.98$ & $2.96 \pm 2.27$ & $0.116^{* *}$ \\
\hline APACHEII score & $14.35 \pm 5.2$ & $12.41 \pm 4.86$ & $14.2 \pm 4.04$ & $15.15 \pm 5.11$ & $0.097 * *$ \\
\hline
\end{tabular}


A. Edalatkhah, et al.

\begin{tabular}{lccccc}
\multicolumn{2}{l}{ Table 2. Comparison of mortality rate at the end of the first, second, fourth and seventh days } & & \\
\hline Mortality & $\begin{array}{c}\text { Etomidate } \\
\mathrm{N}=12\end{array}$ & $\begin{array}{c}\text { Ketamine } \\
\mathrm{N}=8\end{array}$ & $\begin{array}{c}\text { Sodium thiopental } \\
\mathrm{N}=21\end{array}$ & $\begin{array}{c}\text { Midazolam } \\
\mathrm{N}=35\end{array}$ & $\mathrm{p}^{*}$ \\
\hline First day mortality Number (percent) & $3(25 \%)$ & $1(12.5 \%)$ & $3(14.3 \%)$ & $5(14.3 \%)$ & 0.822 \\
$\begin{array}{l}\text { Second day mortality Number (per- } \\
\text { cent) }\end{array}$ & $10(83.3 \%)$ & $1(12.5 \%)$ & $6(28.6 \%)$ & $9(25.7 \%)$ & 0.001 \\
Fourth day mortality Number (percent) & $10(83.3 \%)$ & $1(12.5 \%)$ & $9(42.9 \%)$ & $15(42.9 \%)$ & 0.015 \\
Seven day mortality Number (percent) & $12(100 \%)$ & $2(25 \%)$ & $13(61.9 \%)$ & $23(65.7 \%)$ & 0.007 \\
\hline
\end{tabular}

${ }^{*}$ Chi-square

sure, oxygen saturation, SOFA score, and APACHEII score $(p>0.05)$. However, the heart rate was significantly higher in the etomidate and sodium thiopental groups $(\mathrm{p}<0.001)$ and positive $\mathrm{PMH}$ was significantly prevalent in midazolam and sodium thiopental groups (Table 1).

Based on the data, the etomidate, ketamine, sodium thiopental, and midazolam groups had the mortality rates of $25 \%, 12.5 \%, 14.3 \%$, and $14.3 \%$, respectively, at the end of the first day of intubation $(\mathrm{p}=0.822)$. At the end of the second day, these rates were estimated at $83.3 \%, 12.5 \%$, $28.6 \%$, and $25.7 \%(\mathrm{p}=0.001)$, respectively. Furthermore, the patients in the 4 groups had the mortality rates of $83.3 \%, 12.5 \%, 42.9 \%$, and $42.9 \%(\mathrm{p}=0.015)$, respectively, at the end of the fourth day. These rates were also reported as $100 \%, 25 \%, 61.9 \%$, and $65.7 \%(\mathrm{p}=0.007)$, respectively, at the end of the seventh day (Table 2).

\section{Discussion}

The aim of this study was to compare the effect of etomidate, ketamine, sodium thiopental, and midazolam on the mortality of patients with COVID-19 who needed intubation .In our study, patients with direct laryngoscopy or video laryngoscopy were successfully intubated, and none of them were required to use surgical procedures, suggesting that intubation in patients with COVID-19 was not more difficult than other patients. Also, Aziz et al expressed their experiences gained from the intubation of patients with COVID-19 in Wuhan, China, and reported that $89 \%$ of 202 intubated patients admitted to the center at the first try and $100 \%$ patients without the need for surgical procedures (8).

However, the recent studies reporting some complications during intubation have caused some considerations in this regard. In a study, the mortality of critically ill patients with COVID-19 was reported to be about $10 \%$ in the first 24 hours, and their long-term mortality was $49 \%$ (9); however, this rate underwent a remarkable increase after intubation (almost 89\%) (3). In the present study, the mortality rates were $15.8 \%, 34.2 \%, 46.1 \%$, and $65.8 \%$ until the end of the first, second, fourth, and seventh days, respectively.

Before intubation, $76.3 \%, 42.1 \%$, and $11.8 \%$ of our patients had hypoxia, tachycardia, and hypotension, respectively. Similarly, Yao et al (10) reported hypoxia (oxygen saturation of $<90 \%$ ) and hypotension in $75.2 \%$ and $17.8 \%$ of patients, respectively, before intubation. In addition, in a study performed by Cheung et al, $73 \%$ of the patients suffered from hypoxia, and $80 \%$ of them were intubated without noninvasive ventilation that indicated the progressive course of the disease (11).

In the present study, there was no significant difference among the 4 groups in terms of the mortality rate at the end of the first day. However, at the end of the second, fourth, and seventh days, etomidate groups had a significantly higher mortality rate, compared to other groups. In the hospitals investigated in the present study, there was a strong tendency to use sodium thiopental and midazolam, while in the study by Yao et al (10), the most common drugs were propofol (96\%), midazolam (13.5\%), and etomidate $(3 \%)$, with several patients being administered with 2 drugs at the same time.

The selection of the medication for patient intubation should be made by considering the complications of the drug and patient clinical condition. Etomidate, a gammaaminobutyric acid agonist, is widely used as an induction drug to make anesthesia easier for the patient. This drug can cause adrenal insufficiency by inhibiting 11 betahydroxylase and preventing the conversion of cholesterol into cortisol. Therefore, caution should be exercised when using this medication in cases with septic shock and adrenal insufficiency (12).

In 2019, Smischney et al (13) comparing the composition of propofol/ketamine with etomidate for the intubation of 152 critically ill patients, found no significant difference between the 2 groups in terms of the mean changes in blood pressure and the need for vasopressor. In the mentioned study, the etomidate group showed more primary adrenal insufficiency than the other group (81\% vs $38 \% ; p=0.027)$. In addition, the ketamine/propofol group was reported to experience 2 life-threatening complications, while the etomidate group had 4 complications.

The admission to intubation time interval was significantly shorter in the etomidate group. This may show the worse clinical condition in the emergency department or faster deterioration of the patients who had been intubated using etomidate or may be due to the greater interest of the emergency specialists of our medical centers in the use of etomidate.

This study has potential limitations. The effect estimates in the model are based on a retrospective observational study. They are, therefore, subject to biases and confounding that may have influenced our model estimates. Small sample size was another important limitation of this study. Due to the pilot nature of the study and the small sample size, the statistical analyses were not very advanced. 


\section{Conclusion}

Based on the results of this study, it can be concluded that the use of etomidate as an induction agent for rapid sequence intubation in patients with COVID-19 can be associated with a higher risk of mortality in these patients. However, the mechanism of this complication is not well understood yet. Further studies are needed to verify the results of this study.

\section{Conflict of Interests}

The authors declare that they have no competing interests.

\section{References}

1. Phua J, Weng L, Ling L, Egi M, Lim CM, Divatia JV, et al. Intensive care management of coronavirus disease 2019 (COVID-19): challenges and recommendations. Lancet Respir Med. 2020 Apr 6.

2. Wang D, Hu B, Hu C, Zhu F, Liu X, Zhang J, et al. Clinical characteristics of 138 hospitalized patients with 2019 novel coronavirus-infected pneumonia in Wuhan, China. JAMA. 2020.

3. Yang X, Yu Y, Xu J, Shu H, Liu H, Wu Y, et al. Clinical course and outcomes of critically ill patients with SARS-CoV-2 pneumonia in Wuhan, China: a singlecentered, retrospective, observational study. Lancet Respir Med. 2020.

4. Arentz M, Yim E, Klaff L, Lokhandwala S, Riedo FX, Chong M, et al. Characteristics and outcomes of 21 critically ill patients with COVID-19 in Washington state. JAMA. 2020.

5. Novel Coronavirus Pneumonia Emergency Response Epidemiology Team. The epidemiological characteristics of an outbreak of 2019 novel coronavirus diseases (COVID-19) in China. Zhonghua Liu Xing Bing Xue Za Zhi. 2020;41:145-51 (in Chinese).

6. Ruan Q, Yang K, Wang W, Jiang L, Song J. Clinical predictors of mortality due to COVID-19 based on an analysis of data of 150 patients from Wuhan, China. Intensive Care Med. 2020.

7. Alhazzani W, Moller MH, Arabi YM, Loeb M, Gong MN, Fan E, et al. Surviving Sepsis Campaign: guidelines on the management of critically ill adults with Coronavirus Disease 2019 (COVID-19). Intensive Care Med. 2020

8. Aziz MF. The Wuhan COVID-19 intubation experience. Br J Anaesth. 2020 Apr 27

9. Wu Z, McGoogan JM. Characteristics of and important lessons from the coronavirus disease 2019 (COVID-19) outbreak in China: summary of a report of 72314 cases from the Chinese Center for Disease Control and Prevention. JAMA. 2020.

10. Yao W, Wang T, Jiang B, Gao F, Wang L, Zheng $\mathrm{H}$, et al. Emergency tracheal intubation in 202 patients with COVID-19 in Wuhan, China: lessons learnt and international expert recommendations. Br J Anaesth. 2020 Apr 10.

11. Cheung JCH, Ho LT, Cheng JV, Cham EYK, Lam KN. Staff safety during emergency airway management for COVIDQ15 19 in Hong Kong. Lancet Respir Med. 2020;8:e19

12. Rocchio MA, Sylvester K, Beik N, Glasser NM, Szumita PM. Retrospective review of propofol versus etomidate during rapid sequence intubation in the emergency department at a tertiary academic center. J. Pharm. Pharmacol. 2017 Jul 17;8(7):231-41.

13. Smischney NJ, Nicholson WT, Brown DR, De Moraes AG, Hoskote SS, Pickering B, et al. Ketamine/propofol admixture vs etomidate for intubation in the critically ill: KEEP PACE Randomized clinical trial. Surg J Trauma Acute Care Surg. 2019 Oct 1;87(4):883-91. 